\title{
On Integrability of the FitzHugh-Rinzel Model
}

\author{
N. A. Kudryashov
}

The integrability of the FitzHugh - Rinzel model is considered. This model is an example of the system of equations having the expansion of the general solution in the Puiseux series with three arbitrary constants. It is shown that the FitzHugh-Rinzel model is not integrable in the general case, but there are two formal first integrals of the system of equations for its description. Exact solutions of the FitzHugh-Rinzel system of equations are given.

Keywords: FitzHugh-Rinzel model, Painlevé test, first integral, general solution, exact solution

\section{Introduction}

One of the most important problems now is understanding how impulses are propagated from one neuron to another. The solution of this problem would not only provide a better understanding of complex processes, but also allow significant progress in the creation of artificial intelligence.

It is well known that the classical standard model in neuroscience is the FitzHugh - Nagumo model. This model was presented in [1-3] as a simplification of the famous Hodgkin-Huxley model [4] for description of the impulse propagation from one neuron to another.

The FitzHugh - Nagumo model has been studied many times in the periodical literature. Some recent results can be found, for example, in [5-12].

There is an important question of the existence of exact solutions for many models in neuroscience. Firstly, we need to obtain analytical dependencies on time for activation functions in constructing neuron networks. Secondly, it is necessary to test different applications of models in neuroscience, and the best way is to check the mathematical model taking into account the exact solutions of nonlinear differential equations. To investigate the question of integrability and to find exact solutions of the nonlinear mathematical model, we can use the Painlevé test.

Received March 03, 2019

Accepted March 17, 2019

This research was supported by the Russian Science Foundation under Grant No 18-11-00209 "Development of methods for investigation of nonlinear mathematical models".

Nikolay A. Kudryashov

nakudr@gmail.com

Department of Applied Mathematics, National Research Nuclear University MEPHI

Kashirskoe sh. 31, Moscow, 115409 Russia

RUSSIAN JOURNAL OF NONLINEAR DYNAMICS, 2019, 15(1), 13-19 
In the recent paper [15], the authors considered the analytical properties of the so-called FitzHugh-Rinzel model and built some simple solutions of the system of equations used to describe the process of neuron propagation. They showed that the general solution of a thirdorder equation is represented by a Puiseux series with three arbitrary constants.

This raises the question of the integrability of a third-order equation that corresponds to this system of equations.

The FitzHugh - Rinzel model is described by the following system of equations [13-15]:

$$
\begin{gathered}
v_{t}=v-\frac{1}{3} v^{3}-w+y+I_{\text {ext }}, \\
w_{t}=\delta(v-\beta w), \\
y_{t}=\mu(\sigma-v-\gamma y) .
\end{gathered}
$$

The system of equations (1.1) can be written in the form of third-order nonlinear ordinary equations at $\beta \delta \neq \mu \gamma$ as follows:

$$
v_{t t t}+\left(v^{2}+a-1\right) v_{t t}+2 v v_{t}^{2}+\left(a v^{2}+c\right) v_{t}+\frac{1}{3} b v^{3}+e v-\left(I_{e x t}+\sigma\right) \beta \mu \delta=0,
$$

where

$$
a=\beta \delta+\gamma \mu, \quad b=\beta \delta \gamma \mu, \quad c=b-a+\delta+\mu, \quad e=\mu \delta(\beta+\gamma)-b .
$$

The origin of the expansion for the general solution of the system of equations (1.1) with three arbitrary constants in the Puiseux series is explained by the fact that two of the three equations of the system are linear and therefore by definition do not have critical movable singular points. The first-order equation of the system (1.1) is nonlinear, but it always has a critical pole at $t=t_{0}$ with the exception of quadratic nonlinearity.

The aim of this note is to consider the first integrals of the third-order differential equation (1.2) and to find the general solution of this equation taking into account the values of the Fuchs indices at the expansion of the general solution in the Puiseux series.

\section{First integrals of the FitzHugh-Rinzel model}

The first term of the expansion of the general solution in the Puiseux series can be found from the equation with the leading terms

$$
v_{t t t}+v^{2} v_{t t}+2 v v_{t}^{2}=\frac{d^{2}}{d t^{2}}\left(v_{t}+\frac{1}{3} v^{3}\right)=0 .
$$

We can note that the equation with leading terms is determined by calculating the second derivative of two terms of the first equation (1.1). We can see that Eq. (1.2) does not pass the Painlevé test because there is the critical movable point in the expansion [16-21]. However, there is the expansion of the general solution of Eq. (1.2) in the Puiseux series with three arbitrary constants $t_{0}, a_{3}$ and $a_{5}$ in the form [15]

$$
\begin{aligned}
v(t) & = \pm \frac{\sqrt{6}}{2 \sqrt{t-t_{0}}} \pm \frac{\sqrt{6}}{4} \sqrt{t-t_{0}} \mp \\
& \mp a_{3}\left(t-t_{0}\right) \pm \frac{\sqrt{6}}{48}(1+16 b-16 a-16 c)\left(t-t_{0}\right)^{3 / 2} \mp a_{5}\left(t-t_{0}\right)^{2} \ldots
\end{aligned}
$$


Let us note that we have a critical movable pole of the general solution and the three arbitrary constants $t_{0}, a_{3}$ and $a_{5}$ for the expansion of the general solution in the Puiseux series. These arbitrary constants may correspond to first integrals with the leading terms of $v_{t}+m v^{3}$ and $v_{t t}+m v^{2} v_{t}[22,23]$.

With these observations in mind, we can look for the first integrals for Eq. (1.2) in the form

$$
v_{t t}=S(t)-v^{2} v_{t}-m v^{3}-n v_{t}-p v,
$$

where the coefficients $m, n, p$ and the function $S(t)$ should be determined from the compatibility conditions with Eq. (1.2).

Differentiating Eq. (2.3) with respect to $t$ and substituting $V_{t t t}$ and $v_{t t}$ into Eq. (1.2), we can find parameters $m, n$ and $p$ in the form

$$
\begin{aligned}
& m_{1}=\beta \delta-1, \quad n_{1}=\frac{1}{3} \beta \delta, \quad p_{1}=\delta+\mu-\beta \delta, \\
& m_{2}=\gamma \mu-1, \quad n_{2}=\frac{1}{3} \gamma \mu, \quad p_{2}=\delta+\mu-\gamma \mu .
\end{aligned}
$$

To obtain the equation for the function $S(t)$, we have to take into account the additional condition for parameters $\beta, \delta \gamma$ and $\mu$ of the third-order differential equation (1.2) in the form

$$
\mu(\beta \delta-\gamma \mu)=0
$$

In this case we get the equation for the function $S(t)$ in the form

$$
S_{t}+\beta \delta S=0
$$

At $\mu=0$ and $\beta \delta \neq \mu \gamma$ we obtain a first integral in the form

$$
v_{t t}+v^{2} v_{t}+(\beta \delta-1) u_{t}+\frac{1}{3} u^{3}+\delta(1-\beta) u=C_{1} .
$$

This first integral corresponds to the partial case of Eq. (1.2) at $\mu=0$

$$
v_{t t t}+\left(v^{2}+\beta \delta-1\right) v_{t t}+2 v v_{t}^{2}+\left(\beta \delta u^{2}-\beta \delta+\delta\right) u_{t}=0 .
$$

Let us forget how Eq. (1.2) was obtained, then the first integral of Eq. (1.2) at $\mu=\frac{\beta \delta}{\gamma}$ takes the form

$$
v_{t t}+v^{2} v_{t}+2 v v_{t}^{2}+(\beta \delta-1) v_{t}+\frac{\beta \delta}{3} v^{3}+\delta\left(1+\frac{\beta}{\gamma}-\beta\right) v=C_{1} e^{-\beta \delta t} .
$$

This integral is also a special case of Eq. (1.2)

$$
\begin{gathered}
v_{t t t}+\left(v^{2}+\beta \delta-1\right) v_{t}+2 v v_{t}^{2}+\beta \delta\left(2 v^{2}+2 \beta \delta-2+\frac{1}{\beta}+\frac{1}{\gamma}\right) v_{t}+ \\
+\frac{\beta^{2} \delta^{2}}{3} v^{3}+\beta^{2} \delta^{2}\left(\frac{1}{\beta}+\frac{1}{\gamma}-1\right) v=0 .
\end{gathered}
$$

In the case $\delta=0$ the system of equations is transformed into the system of two equations. At $\delta \neq 0$ we have $\gamma=-\beta$ and the first integral of Eq. (2.9) can be written as

$$
v_{t}+\frac{1}{3} v^{3}-v=\left(C_{2}+C_{1} t\right) e^{-\beta \delta t}
$$


Equation (1.2) at $\gamma=-\beta$ and $\mu=-\delta$ takes the form

$$
v_{t t t}+\left(v^{2}+\beta \delta-1\right) v_{t}+2 v v_{t}^{2}+2 \beta \delta\left(v^{2}+\beta \delta-1\right) v_{t}+\frac{\beta^{2} \delta^{2}}{3} v^{3}-\beta^{2} \delta^{2} v=0 .
$$

The Cauchy problem for this equation can be solved using Eq. (2.11) with two arbitrary constants $C_{1}$ and $C_{2}$.

Let us consider the case $\mu=\frac{\beta \delta}{\gamma}$ using the system of equations (1.1). Substituting this condition into the system of equations (1.1), we have a system in the form

$$
\begin{gathered}
v_{t}=v-\frac{1}{3} v^{3}-w+y+I_{\text {ext }}, \\
w_{t}+\delta \beta w=\delta v, \\
y_{t}+\beta \delta y=\frac{\beta \delta \sigma}{\gamma} .
\end{gathered}
$$

Substituting $y-w$ from the first equation of the system of equations (2.13) into the difference of the two last Eqs. (2.13), we have a second-order differential equation in the form

$$
v_{t t}+v^{2} v_{t}+\beta \delta v_{t}-v_{t}-\beta \delta v-\beta \delta I_{e x t}+\frac{1}{3} \beta \delta v^{3}+\frac{\beta \delta}{\gamma} v_{t}-\frac{\beta \delta \sigma}{\gamma}=0 .
$$

Taking into account the Fuchs index equal to $\frac{3}{2}$, we obtain a first integral in the form

$$
v_{t}+\frac{1}{3} v^{3}-v=I_{e x t}+\frac{\sigma}{\beta}+C_{2} e^{-\beta \delta t}
$$

Let us note that the first integral (2.15) coincides with the first integral of the third-order differential equation (1.2) at $C_{1}=0$. These first integrals can be used for construction of exact solutions for the system of equations (1.1).

\section{Exact solutions of the FitzHugh-Rinzel model}

It seems to us that the general solution of the nonautonomous first-order differential equation (2.11) cannot be found. We could not find a general solution of Eq. (2.11) in the handbooks [24-26]. Naturally, the general solution of the equation at $C_{1} \neq 0$ and $C_{2} \neq 0$ cannot be found by the conventional methods presented, for the example, in [27-31].

At $C_{1}=C_{2}=0$ we have the Bernoulli equation from (2.11) in the form

$$
v_{t}+\frac{1}{3} v^{3}-v=0
$$

which is linearized by means of transformations

$$
v(t)=\frac{\sqrt{3}}{2 \omega(t)^{1 / 2}} .
$$

As a result, we have the equation

$$
\omega_{t}+2 \omega=1
$$


with the solution

$$
\omega(t)=1+C_{1} e^{-2 t} .
$$

Taking into account formula (3.2), we get a solution for $u(t)$ in the form

$$
v(t)=\frac{\sqrt{3}}{\sqrt{1+3 C_{1} e^{-2 t}}} .
$$

Assuming $C_{1}=0$ and $C_{2} \neq 0$, we get an equation from (2.11) in the form

$$
v_{t}+\frac{1}{3} v^{3}-v=C_{2} e^{-\beta \delta t}
$$

Let us look for a solution of Eq. (3.6) in the form

$$
v(t)=w(t) e^{t} .
$$

Substituting expression (3.7) into (3.6), we obtain

$$
w_{t} e^{t}+\frac{1}{3} w^{3} e^{3 t}=C_{2} e^{-\beta \delta t} .
$$

The last equation is transformed at $\beta \delta=-3$ to the following integral:

$$
w(t)=\left(3 C_{2}\right)^{1 / 3} F(t), \quad \int \frac{d F}{1-F^{3}}=C_{3}+\frac{C_{2}}{2\left(3 C_{2}\right)^{1 / 3}} e^{2 t} .
$$

Calculating integral (3.9) with respect to $F$, we have a solution in the form

$$
v(t)=e^{t} w(t)=e^{t}\left(3 C_{2}\right)^{1 / 3} F(t),
$$

where the function $F(t)$ is determined from the equation

$$
\ln \left[\frac{F^{2}+F+1}{F^{2}-2 F+1}\right]+2 \sqrt{3} \arctan \left[\frac{\sqrt{3}}{3}(2 F+1)\right]=6 C_{3}+\frac{6 C_{2}}{2\left(3 C_{2}\right)^{1 / 3}} e^{2 t} .
$$

The solution of the FitzHugh - Rinzel system of equations (3.9) has two arbitrary constants.

The authors of [15] considered the same dependence of $t$ for functions $y(t)$ and $w(t)$ in the form

$$
y=w-\frac{\sigma}{\beta} .
$$

In this case there are two equations in the form

$$
\begin{gathered}
v_{t}+\frac{1}{3} v^{3}-v=0, \\
w_{t}+\beta \gamma=\delta v .
\end{gathered}
$$

The first equation of this system is linearized as we have shown before, and the second equation of the system is the first-order linear equation. However, this integrable case without the Painlevé property corresponds to the second-order differential equation of the FitzHugh-Rinzel system of equations. 


\section{Conclusion}

In this note we have considered the FitzHugh-Rinzel model for description of the propagation from one neuron to another. This topic was studied in the paper [15], and this note has been written under the influence of this article.

The main interest in this system of equations is due to the fact that the general solution of this system, due to the specificity of the system, has an expansion of the general solution in the Puiseux series with three arbitrary constants. In fact, there is a local general (though nonmeromorphic) solution to this system of equations. This raises the question of the global solution and the existence of first integrals for the third-order equation obtained from the FitzHugh - Rinzel system of equations.

In this note we have shown that there are two nonautonomous first integrals for the thirdorder differential equation corresponding to the FitzHugh-Rinzel system of equations which allow us to obtain some exact solutions of this system.

\section{References}

[1] FitzHugh, R., Impulses and Physiological States in Theoretical Models of Nerve Membrane, Biophys. J., 1961, vol. 1, no. 6, pp. 445-466.

[2] Nagumo, J., Arimoto, S., and Yoshizawa, S., An Active Pulse Transmission Line Simulating Nerve Axon, Proc. of the IRE, 1962, vol. 50, no. 10, pp. 2061-2070.

[3] FitzHugh, R., A Kinetic Model for the Conductance Changes in Nerve Membranes, J. Cell. Comp. Physiol., 1965, vol. 66, suppl. 2, pp. 111-117.

[4] Hodgkin, A. L. and Huxley, A. F., A Quantitative Description of Membrane Current and Its Application to Conduction and Excitation in Nerve, J. Physiol., 1952, vol. 117, no. 4, pp. 500-544.

[5] Postnikov, E. B. and Titkova, O. V., A Correspondence between the Models of Hodgkin-Huxley and FitzHugh-Nagumo Revised, Eur. Phys. J. Plus, 2016, vol.131, no. 11, Art. 411.

[6] Saha, A. and Feudel, U., Extreme Events in FitzHugh - Nagumo Oscillators Coupled with Two Time Delays, Phys. Rev. E, 2017, vol. 95, no. 6, 062219, 10 pp.

[7] Schmidt, A., Kasmatis, Th., Hizanidas, J., Provata, A., and Hövel, P., Chimera Patterns in TwoDimensional Networks of Coupled Neurons, Phys. Rev. E, 2017, vol. 95, no. 3, 032224, 13 pp.

[8] Zemskov, E.P., Tsyganov, M.A., and Horsthemke, W., Oscillatory Pulses and Wave Trains in a Bistable Reaction-Diffusion System with Cross Diffusion, Phys. Rev. E, 2017, vol.95, no.1, 012203, 9 pp.

[9] Scott, A., Nonlinear Science. Emergence and Dynamics of Coherent Structures, 2nd ed., Oxford: Oxford Univ. Press, 2005.

[10] Kudryashov, N.A., Asymptotic and Exact Solutions of the FitzHugh-Nagumo Model, Regul. Chaotic Dyn., 2018, vol.23, no. 2, pp. 152-160.

[11] Kudryashov, N.A., Rybka, R.B., and Sboev, A. G., Analytical Properties of the Perturbed FitzHugh-Nagumo Model, Appl. Math. Lett., 2018, vol. 76, pp. 142-147.

[12] Llibre, J. and Vidal, C., Periodic Solutions of a Periodic FitzHugh-Nagumo System, Internat. J. Bifur. Chaos Appl. Sci. Engrg., 2015, vol. 25, no. 13, 1550180, 6 pp.

[13] Rinzel, J., A Formal Classification of Bursting Mechanisms in Excitable Systems, in Mathematical Topics in Population Biology, Morphogenesis and Neurosciences, E. Teramoto, M. Yamaguti (Eds.), Lecture Notes in Biomath., vol. 71, Berlin: Springer, 1987, pp. 267-281.

[14] Belykh, V.N. and Pankratova, E. V., Chaotic Synchronization in Ensembles of Coupled Neurons Modeled by the FitzHugh - Rinzel System, Radiophys. Quantum El., 2006, vol. 49, no. 11, pp. 910921; see also: Izv. Vyssh. Uchebn. Zaved. Radiofizika, 2006, vol. 49, no. 11, pp. 1002-1014. 
[15] Zemlyanukhin, A.I. and Bochkarev, A.V., Analytical Properties and Solutions of the FitzHughRinzel Model, Russian J. Nonlinear Dyn., 2019, vol.15, no. 1, pp. 3-12.

[16] Painlevé, P., Sur les équations différentielles du second ordre et d'ordre supérieur dont l'intégrale générale est uniforme, Acta Math., 1902, vol. 25, pp. 1-85.

[17] Gambier, B., Sur les équations différetielles dont l'integrate générale est uniforme, C. R. Acad. Sci. Paris, 1906, vol.142, pp. 266-269, 1403-1406, 1497-1500.

[18] Kudryashov, N. A., Amalgamations of the Painlevé Equations, J. Math. Phys., 2003, vol. 44, no. 12, pp. 6160-6178.

[19] Borisov, A. V. and Kudryashov, N. A., Paul Painlevé and His Contribution to Science, Regul. Chaotic Dyn., 2014, vol. 19, no. 1, pp.1-19.

[20] Kudryashov, N. A., Higher Painlevé Transcensents As Special Solutions of Some Nonlinear Integrable Hierarchies, Regul. Chaotic Dyn., 2014, vol.19, no. 1, pp. 48-63.

[21] Kudryashov, N. A., Nonlinear Differential Equations Associated with the First Painlevé Hierarchy, Appl. Math. Lett., 2019, vol.90, pp. 223-228.

[22] Kudryashov, N. A., Exact Solutions of the Equation for Surface Waves in a Convecting Fluid, Appl. Math. Comput., 2019, vol. 344/345, pp. 97-106.

[23] Kudryashov, N. A., Exact Solutions and Integrability of the Duffing-van der Pol Equation, Regul. Chaotic Dyn., 2018, vol. 23, no. 4, pp. 471-479.

[24] Polyanin, A. D. and Zaitsev, V.F., Handbook of Exact Solutions for Ordinary Differential Equations, 2nd ed., Boca Raton, Fla.: Chapman \& Hall/CRC, 2002.

[25] Polyanin, A. D. and Zaitsev, V.F., Handbook of Nonlinear Partial Differential Equations, 2nd ed., Boca Raton, Fla.: CRC, 2012.

[26] Polyanin, A. D. and Zaitsev, V.F., Handbook of Ordinary Differential Equations: Exact Solutions, Methods, and Problems, Boca Raton, Fla.: Chapman \& Hall/CRC, 2017.

[27] Kudryashov, N.A., Exact Solutions of the Generalized Kuramoto-Sivashinsky Equation, Phys. Lett. A, 1990, vol. 147, nos. 5-6, pp. 287-291.

[28] Kudryashov, N. A., Solitary and Periodic Solutions of the Generalized Kuramoto-Sivashinsky Equation, Regul. Chaotic Dyn., 2008, vol. 13, no.3, pp. 234-238.

[29] Kudryashov, N. A., One Method for Finding Exact Solutions of Nonlinear Differential Equations, Commun. Nonlinear Sci. Numer. Simul., 2012, vol.17, no. 6, pp. 2248-2253.

[30] Kudryashov, N. A., Polynomials in Logistic Function and Solitary Waves of Nonlinear Differential Equations, Appl. Math. Comput., 2013, vol. 219, no. 17, pp. 9245-9253.

[31] Kudryashov, N.A., Painlevé Analysis and Exact Solutions of the Korteweg-de Vries Equation with a Source, Appl. Math. Lett., 2015, vol.41, pp. 41-45. 